\title{
General properties of logarithmically divergent one-loop lattice Feynman integrals
}

\author{
Jongjeong Kim* \\ Department of Physics and Astronomy, Seoul National University, Seoul, 151-747, South Korea \\ E-mail: rvanguardephya.snu.ac.kr
}

\section{David H. Adams}

Department of Physics and Astronomy, Seoul National University, Seoul, 151-747, South Korea

E-mail: dadams@phya.snu.ac.kr

\section{Weonjong Lee}

Frontier Physics Research Division and Center for Theoretical Physics,

Department of Physics and Astronomy, Seoul National University, Seoul, 151-747, South Korea

E-mail: wlee@phya.snu.ac.kr

\begin{abstract}
We prove that logarithmically divergent one-loop lattice Feynman integrals have the general form $I(p, a)=f(p) \log (a M)+g(p, M)$ up to terms which vanish for lattice spacing $a \rightarrow 0$. Here $p$ denotes collectively the external momenta and $M$ is an arbitrary mass scale. The $f(p)$ is shown to be universal and to coincide with the analogous quantity in the corresponding continuum integral (regularized, e.g., by momentum cut-off). This is essential for universality of the lattice QCD beta-function and anomalous dimensions of renormalized lattice operators at one loop. The result and argument presented here are simplified versions of ones given in arXiv:0709.0781. A noteworthy feature of the argument here is that it does not involve Taylor expansion in external momenta, hence infra-red divergences associated with that expansion do not arise.
\end{abstract}

The XXV International Symposium on Lattice Field Theory

July 30-4 August 2007

Regensburg, Germany

\footnotetext{
${ }^{*}$ Speaker.
} 


\section{Introduction}

Lattice folklore asserts that the one-loop beta-function and anomalous dimensions of one-loop renormalized operators are universal in lattice formulations of QCD and coincide with the corresponding continuum quantities. While this is certainly the case for the explicit lattice formulations considered to date, no general proof for general lattice QCD formulations has been given previously. The renormalization factors that one needs to consider in connection with this are given by logarithmically divergent one-loop lattice Feynman integrals $I(p, a)$. In this paper we derive a general structural result for such integrals which provides a basis for proving the mentioned folklore assertions. We show that these integrals have the general structure

$$
I(p, a)=f(p) \log (a M)+g(p, M)
$$

up to terms which vanish for lattice spacing $a \rightarrow 0$. Here $p$ denotes collectively the external momenta and $M$ is a mass scale which may be chosen arbitrarily. The key features are (i) the lattice spacing dependence enters exclusively through the $\log (a M)$ term, and (ii) the factor $f(p)$ can be expressed as a convergent continuum integral and is therefore universal; moreover,

$$
f(p)=f^{(c)}(p)
$$

where $f^{(c)}(p)$ is the analogous factor in the corresponding continuum integral, which has the structure

$$
I^{(c)}(p, \Lambda)=f^{(c)}(p) \log (M / \Lambda)+g^{(c)}(p, M)
$$

when regularized by a momentum cut-off $\Lambda$. (Analogous statements hold when dimensional regularization is used; see Ref.[1].)

A stronger version of the structural results (1.1)-(1.2) was recently proved in Ref.[1]. ${ }^{12}$ The argument there gave more information on the structure of $g(p, M)$ in (1.1). However, (1.1)-(1.2) alone can be established by a simpler argument than the one given there, which we will present here. In particular, the argument avoids the usual procedure of separating off the leading term in the expansion of $I(p, a)$ in $p$ and having to deal with its infra-red divergence.

The argument proceeds in two main steps: First we define

$$
f(p):=\lim _{a \rightarrow 0} a \frac{d}{d a} I(p, a)
$$

and show that it is finite and given by a convergent continuum integral coinciding with $f^{(c)}(p)$. Next we define

$$
g(p, M):=\lim _{a \rightarrow 0}(I(p, a)-f(p) \log (a M))
$$

and prove that it is finite. Together these imply the structural results (1.1)-(1.2).

\footnotetext{
${ }^{1}$ This structure was expected [2,3] but we emphasize that no explicit general proof had previously been given.

${ }^{2}$ Some of the techniques and results of Ref.[1] were developed earlier in a special case in Ref.[4]
} 


\section{The setup}

One-loop lattice Feynman integrals have the following general form:

$$
\begin{gathered}
I(p, a)=\int_{-\pi / a}^{\pi / a} d^{4} k \frac{V(k, p, a)}{C(k, p, a)} \\
V(k, p, a)=\frac{1}{a^{m}} F(a k, a p) \quad, \quad C(k, p, a)=\frac{1}{a^{n}} G(a k, a p)
\end{gathered}
$$

The lattice degree [5] of $V(k, p, a)$ in $k$ can be characterized as follows. Let $r$ denote the order of the first non-vanishing term in the $t$-expansion of $F(k, t p)$ around $t=0$,

$$
F(k, t p)=t^{r} F_{0}(k, p)+t^{r+1} F_{1}(k, p, t)
$$

Then $V\left(\lambda k, p, \frac{a}{\lambda}\right)=\left(\frac{\lambda}{a}\right)^{m} F\left(a k, \frac{a}{\lambda} p\right) \sim \lambda^{m-r}$ for $\lambda \rightarrow \infty$, hence the lattice degree of $V$ is $\hat{d}_{V}=m-r$ [5].

We assume that $C(k, p, a)$ arises as a product of lattice propagators as described in Reisz's work [5]. Then the expansion of $G(k, t p)$ in $t$ has non-vanishing zero-order term:

$$
G(k, t p)=G(k, 0)+t G_{1}(k, p, t)
$$

hence $C\left(\lambda k, p, \frac{a}{\lambda}\right)=\left(\frac{\lambda}{a}\right)^{n} G\left(a k, \frac{a}{\lambda} p\right) \sim \lambda^{n}$ for $\lambda \rightarrow \infty$, so the lattice degree of $C$ is $\hat{d}_{C}=n$. The divergence degree of the lattice integral is then given by [5]:

$$
\hat{d}_{I}=4+\hat{d}_{V}-\hat{d}_{C}=4+m-r-n .
$$

$V(k, p, a)$ and $C(k, p, a)$ are assumed to have finite continuum limits:

$$
V(k, p, a) \stackrel{a \rightarrow 0}{\longrightarrow} P(k, p) \quad, \quad C(k, p, a) \stackrel{a \rightarrow 0}{\longrightarrow} E(k, p)
$$

Then, in light of (2.2), it follows that $P(k, p)$ and $E(k, p)$ are homogeneous polynomials in $(k, p)$ of degrees $m$ and $n$, respectively. We denote the usual degree of $P(k, p)$ in $k$ by $d_{P}$, with $d_{E}$ defined analogously for $E(k, p)$. Note that $d_{P} \leq \hat{d}_{V}$ while $d_{E}=n=\hat{d}_{C}$ [5].

We henceforth specialize to the logarithmically divergent case, i.e., $\hat{d}_{I}=0$, and proceed to carry out the two steps discussed in the introduction for establishing the claimed structural results for $I(p, a)$.

\section{Step 1}

Changing variables to $\hat{k}=a k$ in (2.1) and using $\hat{d}_{I}=4+m-r-n=0$ we find

$$
I(p, a)=\int_{-\pi}^{\pi} d^{4} \hat{k} \frac{a^{-r} F(\hat{k}, a p)}{G(\hat{k}, a p)}
$$

and consequently

$$
\begin{aligned}
a \frac{d}{d a} I(p, a) & =\int_{-\pi}^{\pi} d^{4} \hat{k} a \frac{d}{d a}\left(\frac{a^{-r} F(\hat{k}, a p)}{G(\hat{k}, a p)}\right) \\
& =\int_{-\pi}^{\pi} d^{4} \hat{k} \frac{d}{d t}\left(\frac{(t a)^{-r} F(\hat{k}, t a p)}{G(\hat{k}, t a p)}\right)_{t=1} \\
& =\int_{-\pi / a}^{\pi / a} d^{4} k \frac{d}{d t}\left(\frac{t^{-r} V(k, t p, a)}{C(k, t p, a)}\right)_{t=1}
\end{aligned}
$$


We are going to show that the last integral has lattice divergence degree $\leq \hat{d}_{I}-1=-1$, which will allow us to apply to it the lattice power-counting theorem of Ref.[5]. This is an immediate consequence of the following:

(A) $\frac{d}{d t}\left(t^{-r} V(k, t p, a)\right)$ has divergence degree $\leq \hat{d}_{V}-1$.

(B) $\frac{d}{d t} C(k, t p, a)$ has divergence degree $\leq d_{C}-1$

Proof of (A): Using (2.2)-(2.3) we have

$$
\begin{aligned}
t^{-r} V\left(\lambda k, t p, \frac{a}{\lambda}\right) & =\left(\frac{\lambda}{a}\right)^{m} t^{-r} F\left(a k, \frac{t}{\lambda} a p\right) \\
& =\left(\frac{\lambda}{a}\right)^{m} t^{-r}\left(\left(\frac{t}{\lambda}\right)^{r} F_{0}(a k, a p)+\left(\frac{t}{\lambda}\right)^{r+1} F_{1}\left(a k, a p, \frac{t}{\lambda}\right)\right)
\end{aligned}
$$

The $t$-dependence cancels out in the first term so it vanishes under $\frac{d}{d t}$. Hence the $\lambda \rightarrow \infty$ behavior is determined by the second term, which is $\sim \lambda^{m-r-1}$ or slower.

Proof of $(B)$ : This follows by a similar argument, using (2.4) to write

$$
C\left(\lambda k, t p, \frac{a}{\lambda}\right)=\left(\frac{\lambda}{a}\right)^{n}\left(G(a k, 0)+\frac{t}{\lambda} G_{1}\left(a k, a p, \frac{t}{\lambda}\right)\right)
$$

and noting that the leading term is again $t$-independent and hence vanishes under $\frac{d}{d t}$.

Having established that the lattice integral expression (3.2) for $a \frac{d}{d a} I(p, a)$ has lattice divergence degree $\leq-1$ we can invoke Reisz's lattice power-counting theorem [5] to conclude that its $a \rightarrow 0$ limit is finite and given by the corresponding continuum integral:

$$
f(p):=\lim _{a \rightarrow 0} a \frac{d}{d a} I(p, a)=\int_{-\infty}^{\infty} d^{4} k \frac{d}{d t}\left(\frac{t^{-r} P(k, t p)}{E(k, t p)}\right)_{t=1}
$$

Thus $f(p)$ is given by a convergent continuum integral and hence is universal as claimed. ${ }^{3}$ It remains to show $f(p)=f^{(c)}(p)$. From (1.3) we have

$$
f^{(c)}(p)=\lim _{\Lambda \rightarrow \infty}-\Lambda \frac{d}{d \Lambda} I^{(c)}(p, \Lambda)
$$

Changing integration variable to $\hat{k}=k / \Lambda$ in the continuum integral we find

$$
I^{(c)}(p, \Lambda)=\int_{-\Lambda}^{\Lambda} d^{4} k \frac{P(k, p)}{E(k, p)}=\int_{-1}^{1} d^{4} \hat{k} \frac{\Lambda^{r} P(\hat{k}, p / \Lambda)}{E(\hat{k}, p / \Lambda)}
$$

where we have used that the previously mentioned homogeneity of $P(k, p)$ and $E(k, p)$ in $(k, p)$ implies $P(\Lambda \hat{k}, p)=\Lambda^{m} P(\hat{k}, p / \Lambda)$ and $E(\Lambda \hat{k}, p)=\Lambda^{n} E(\hat{k}, p / \Lambda)$, and also used $0=\hat{d}_{I}=4+m-r-$ $n$. It follows that

$$
\begin{aligned}
-\Lambda \frac{d}{d \Lambda} I^{(c)}(p, \Lambda) & =\int_{-1}^{1} d^{4} \hat{k} \frac{d}{d t}\left(\frac{(\Lambda / t)^{r} P(\hat{k}, p t / \Lambda)}{E(\hat{k}, p t / \Lambda)}\right)_{t=1} \\
& =\int_{-\Lambda}^{\Lambda} d^{4} k \frac{d}{d t}\left(\frac{t^{-r} P(k, t p)}{E(k, t p)}\right)_{t=1}
\end{aligned}
$$

This reduces in the $\Lambda \rightarrow \infty$ limit to our previous expression (3.5) for $f(p)$, thereby showing that

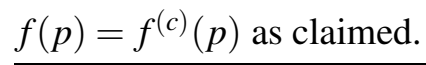

\footnotetext{
${ }^{3}$ A simpler continuum integral expression for $f(p)$ is obtained in Eq.(4.7) of Ref.[1]. It would be better to use that one rather than the present one for evaluating $f(p)$ in practice.
} 


\section{Step 2}

Our goal now is prove that the limit (1.5) is finite. To this end we set

$$
\hat{a}=a M
$$

and define

$$
f(p, \hat{a}):=a \frac{d}{d a} I(p, a)=\hat{a} \frac{d}{d \hat{a}} I(p, \hat{a} / M)
$$

which has $f(p)$ as its $\hat{a} \rightarrow 0$ limit, cf. $\S 3$. Then the quantity in (1.5) whose limit we need to consider can be expressed as

$$
\begin{aligned}
I(p, a)-f(p) \log (a M) & =I(p, \hat{a} / M)-f(p) \log (\hat{a}) \\
& =-\int_{\hat{a}}^{1} d b \frac{1}{b}(f(p, b)-f(p))+I(p, 1 / M)
\end{aligned}
$$

To show that this has finite $a \rightarrow 0$ limit, or equivalently, finite $\hat{a} \rightarrow 0$ limit, we need to show that the integral on the right-hand side remains finite for $\hat{a} \rightarrow 0$. For this we need information on how quickly $f(p, b)$ approaches its continuum limit $f(p)$ for $b \rightarrow 0$. To obtain this, we note that $f(p, \hat{a})$ can be expressed as a convergent lattice integral: Changing variables to $\hat{k}=k / M$ in (3.2) we find, using (2.2) and $\hat{d}_{I}=4+m-r-n=0$,

$$
f(p, \hat{a})=\int_{-\pi / \hat{a}}^{\pi / \hat{a}} d^{4} \hat{k} \frac{d}{d t}\left(\frac{(t / M)^{-r} V(\hat{k}, t p / M, \hat{a})}{C(\hat{k}, t p / M, \hat{a})}\right)_{t=1}
$$

which is seen to have divergence degree $\leq-1$ by the same argument as for (3.2). An extension of the lattice power-counting theorem proved in Ref.[1] now tells that $f(p, \hat{a})-f(p)$ vanishes at least as fast as $\sim \hat{a} \log (1 / \hat{a})$ for $\hat{a} \rightarrow 0$. Applying this with $\hat{a}$ replaced by $b$ allows us to conclude that the integral

$$
\int_{\hat{a}}^{1} d b \frac{1}{b}|f(p, b)-f(p)|
$$

remains finite in the $\hat{a} \rightarrow 0$ limit. ${ }^{4}$ By Lebesgue's "theorem of dominated convergence" the integral continues to have a well-defined finite limit when the integrand is replaced by $\frac{1}{b}(f(p, b)-f(p))$. Hence the $a \rightarrow 0$ limit of (4.3) is finite, i.e., $g(p, M)$ in (1.5) is finite as claimed. This completes the derivation of the structural results (1.1)-(1.2).

\section{Concluding remarks}

Besides depending on the external momenta $p$, the lattice integral $I(p, a)$ may also depend on masses (e.g., fermion masses) if the Feynman diagram involves massive propagators. The dependence of $I(p, a)$ on such masses, which we denote collectively by $m$, is easily described. Their presence in the lattice integrand can be described by replacing $F(a k, a p) \rightarrow F(a k, a p, a m)$ and $G(a k, a p) \rightarrow G(a k, a p, a m)$ in (2.2), and from this it is clear that the $m$-dependence in the structural

\footnotetext{
${ }^{4}$ Note that $\frac{1}{b}(b \log (1 / b))=-\log (b)=\frac{d}{d b}(-b \log (b)+b)$.
} 
results (1.1)-(1.2) can be indicated by replacing $p \rightarrow(p, m)$ in the expressions there. Or we can simply take $p$ to denote collectively the external momenta and masses.

The structural results (1.1)-(1.2) immediately imply universality of the anomalous dimensions of one-loop renormalized lattice operators in the usual case where the renormalization factor is given by a logarithmically divergent lattice Feynman integral. Universality of the one-loop beta-function in lattice QCD requires a bit more elaboration since the individual lattice Feynman diagrams relevant for computing the lattice beta-function can have stronger divergences (linear and quadratic). However, these can be combined into logarithmically divergent integrals by exploiting lattice BRST symmetry and lattice hypercubic symmetries [7, 8], after which the structural results here can be applied. Or we can chose to define the renormalized coupling via the 4-gluon vertex; then, since the one-loop corrections to this vertex in lattice QCD are all either logarithmically divergent or finite, our structural results can be immediately applied. This will be discussed explicitly in a forthcoming publication.

The general conditions under which the structural results here are derived can be summarized by saying that they are the same as the ones in Reisz's work on the lattice power-counting theorem [5] (but with $\hat{d}_{I}=0$ rather than $\hat{d}_{I}<0$ ), except that we do not need a certain technical condition on the lattice propagators that he required. ${ }^{5}$ This is because it was possible to prove the (extended) lattice power-counting theorem in the one-loop case in Ref.[1] without invoking this condition (see [1] for further discussion). To deal with the possibility of infrared divergences when the lattice propagators are massless, Reisz introduced in Ref.[6] the notion of infrared lattice divergence degree and proved infrared finiteness when this degree is strictly negative. We also require this condition here in the case where the lattice propagators are massless. In practice, for the present one-loop case, it usually means that the external momenta $p$ cannot all be zero.

Finally, as a guide for attempting to extend the structural results to divergent multi-loop lattice integrals with general divergence degree, we note that the general structure is expected to be as follows $[2,3]$ :

$$
I(p, a)=a^{-\omega} \sum_{n=0}^{\infty} \sum_{m=0}^{l} c_{m n}(p) a^{n}(\log a)^{m}
$$

Here $\omega$ is the divergence degree of the integral and $l$ is the number of loops. In the one-loop case this can be expressed as a generalization of (1.1):

$$
I(p, a)=a^{-\omega}(f(p, a) \log (a)+g(p, a))
$$

where $f(p, a)=f_{0}(p)+f_{1}(p) a+f_{2}(p) a^{2}+\ldots$ and $g(p, a)=g_{0}(p)+g_{1}(p) a+g_{2}(p) a^{2}+\ldots$.

\section{Acknowledgements}

This research is supported by the KICOS international cooperative research program (KICOS

\footnotetext{
${ }^{5}$ The doubler-free condition is required both in Reisz's work and our treatment. This excludes naive and staggered fermions. However, it is nevertheless often possible to apply the results here to lattice integrals involving these. This can be done when symmetries of the integrand allow the integral to be rewritten as $\int_{-\pi / a}^{\pi / a} d^{4} k(\cdots)=N_{t} \int_{-\pi / 2 a}^{\pi / 2 a} d^{4} k(\cdots)$ where $N_{t}$ is the number of tastes ( $=16$ for naive fermions and 4 for staggered fermions). For example, this is possible for the naive or staggered fermion loop contribution to the one-loop vacuum polarization [9].
} 
grant K20711000014-07A0100-01410), by the KRF grant KRF-2006-312-C00497, by the BK21 program of Seoul National University, and by the DOE SciDAC-2 program.

\section{References}

[1] D.H. Adams and W. Lee, arXiv:0709.0781 [hep-lat]

[2] K. Symanzik, Nucl. Phys. B 226 (1983) 187

[3] M. Luscher and P. Weisz, Nucl. Phys. B 266 (1986) 309

[4] D.H. Adams, hep-lat/0602023

[5] T. Reisz, Comm. Math. Phys. 116 (1988) 81.

[6] T. Reisz, Comm. Math. Phys. 116 (1988) 573.

[7] T. Reisz, Nucl. Phys. B 318 (1989) 417.

[8] H. Kawai, R. Nakayama and K. Seo, Nucl. Phys. B 189 (1981) 40.

[9] H.S. Sharatchandra, H.J. Thun and P. Weisz, Nucl. Phys. B 192, 205 (1981). 